Culture et histoire dans l'espace roman

$12 \mid 2014$

Empreintes/emprunts : entre forces de conformisation et forces d'innovation

\title{
Réécriture mythique en poésie : le cas de Pedro Flores
}

\section{Sonia Fernández Hoyos}

\section{(2) OpenEdition}

\section{Journals}

Édition électronique

URL : https://journals.openedition.org/cher/7135

DOI : $10.4000 /$ cher.7135

ISSN : 2803-5992

Éditeur

Presses universitaires de Strasbourg

Édition imprimée

Date de publication : 7 juillet 2014

Pagination : 223-233

ISBN : 978-2-86820-569-8

ISSN : 1968-035X

Référence électronique

Sonia Fernández Hoyos, «Réécriture mythique en poésie : le cas de Pedro Flores », reCHERches [En ligne], 12 | 2014, mis en ligne le 13 décembre 2021, consulté le 15 décembre 2021. URL : http:// journals.openedition.org/cher/7135; DOI : https://doi.org/10.4000/cher.7135

\section{cc) (i) (2)}

Ce(tte) œuvre est mise à disposition selon les termes de la Licence Creative Commons Attribution -

Pas d'Utilisation Commerciale - Partage dans les Mêmes Conditions 4.0 International. 


\title{
Réécriture mythique en poésie: le cas de Pedro Flores
}

\author{
SONIA FERNÁNDEZ Hoyos \\ Université de Lorraine - Metz
}

'acte de raconter des histoires, de les écouter ou de les lire a été défini
comme un désir humain universel. Ces affirmations sont peut-être trop radicales et il ne s'agit pas d'un acte de foi, mais d'illusion. On peut néanmoins analyser le désir de raconter des histoires dans une perspective historique. Les mythes existent en tant que formes précoces de ce désir, et pourtant ils persistent dans une société de plus en plus aliénée. C’est précisément quand une société est plus obsédée par la consommation et la passivité qu'il y a un courant marquant une rupture qui revient aux formes mythiques afin de raconter à nouveau des histoires classiques. Il s'agit de dire le nouveau, comme dans l'étrange commande proposée par Wittgenstein (peu de temps après avoir conclu son célèbre Tractatus..., 1919), l'expression excentrique qui permet d'atteindre la perfection éthique ou la beauté.

Les débats en cours entre tradition et modernité auront une incidence sur l'acte même de raconter à nouveau. Raconter à nouveau des histoires et des mythes classiques est un phénomène très intéressant, bien que pas du tout nouveau, révélant une inestimable quantité d'informations sur la société qui les produit, depuis un point de vue mémoriel et contemporain. Cela signifie également sortir des conventions établies du système dans le domaine de la langue.

Le livre de poèmes avec lequel la maison d'édition espagnole Tragacanto ouvre sa collection de poésie, Donde príncipes y bestias, de Pedro Flores, révise, "raconte» et s'installe dans la variatio des événements transmis par les différentes versions du mythe de Thésée et le Minotaure afin d'ouvrir de

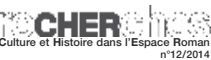


nouvelles interprétations, en se concentrant sur les zones qui étaient plus ou moins restées dans l'ombre dans la transmission précédente.

\section{Le mythe comme genre}

Selon Todorov dans «L'origine des genres» (1987), un genre commence comme un acte de parole et subit différentes transformations avant de devenir une institution. Si nous acceptons ces affirmations, tout est lié au perfectionnement systémique des genres, définis comme des types de textes. Selon ce système, chaque discours se compose d'énoncés et leur signification dépend du contexte dans lequel ils sont produits. Toutefois, accepter ces déclarations signifie utiliser - reconnaître - un meta-discours sur les genres qui a sa propre histoire. En outre, on vise à établir un ensemble de propriétés, de caractéristiques et de normes pour chaque type de texte. Ce type d'approches permet aux critiques de conclure que les genres ne sont que la codification des propriétés discursives. Ces propriétés montrent l'aspect sémantique du texte (sens), l'aspect syntaxique (ou les relations des parties entre elles), l'aspect pragmatique du texte (la relation entre utilisateurs) et l'aspect verbal (tout ce qui concerne la matérialité des signes) (Zipes, 2006: 20). Ces propriétés, qui affectent plusieurs niveaux de texte, devraient être prises en compte au moment de la réception et de la production de nouveaux textes.

Ainsi, les genres - compris comme des institutions - fonctionnent comme des modèles d'écriture pour les auteurs et créent en même temps des horizons d'attente pour les lecteurs. C'est ce processus d'institutionnalisation qui travaille dans une société donnée et qui, dans certaines circonstances, détermine l'existence et les interrelations des genres. L'existence même de certains genres dans une société donnée révèle des informations fort utiles sur l'idéologie sous-jacente.

D'un point de vue diachronique, les mythes (tels que les contes de fées) ont subi de nombreux changements sémantiques. En fait, un changement en particulier identifie les mythes avec les contes de fées dans les registres populaires (Barthes, Bettelheim, Armtrong, Zipes). Cette identification révèle à quel point ces formes ont perdu de leur prestige original parmi d'autres formes littéraires. Il n'est pas surprenant que dans une société encore dominée par les logos scientifiques, les formes littéraires comme celles-ci soient fortement dévaluées. 
Cependant, il y a différents degrés d'importance, une sorte de hiérarchie entre les mythes et les contes de fées. Certains restent dans l'imaginaire collectif, tandis que d'autres sont tombés dans l'oubli. Chaque société sélectionne ses mythes et ses contes conformément à ses conditions et à des attentes particulières, puisque les mythes et les contes de fées forgent une forme appropriée soutenant une idéologie spécifique. C'est la raison pour laquelle on crée un canon concret. Zipes rappelle à cet égard:

[...] estos mitos no son nuevos, ni siquiera son solo mitos, puesto que también son cuentos de hadas. Estos mitos y cuentos de hadas están codificados histórica y culturalmente, y su impacto ideológico es enorme. En cierto modo se han convertido en textos codificados, acreditados y canónicos. Hablamos de mitos clásicos y de cuentos de hadas clásicos. Parece que han estado con nosotros durante siglos, una eternidad, pero perdemos de vista la manera en la que creamos dioses y magia para mantener nuestras experiencias y vidas intactas. (Zipes, 1994: 4) ${ }^{1}$

En effet, ni les mythes ni les contes de fées ne sont des formes éternelles immuables, mais ils doivent être contés à nouveau, reformulés pour se conformer à un moment historique particulier. En outre, le canon est présenté comme une garantie de savoir partagé ou commun, où le contexte pour la même communauté ou société joue un rôle central. Il procure un sentiment de familiarité.

L'apparence d'appartenance devient une sorte de marque dans ces formes. Le canon reproduit la promesse d'une sécurité assurée par un corpus familier. Il implique la reconnaissance et l'identification à un groupe à un moment précis. Toutefois, aucun canon n'est éternel, ils doivent s'adapter aux nouvelles exigences imposées par chaque société. Pour cette raison, certaines œuvres peuvent être intégrées, tandis que d'autres disparaissent.

Les mythes travaillent de telle sorte qu'ils effacent leur évolution historique et se présentent comme des formes a-idéologiques, a-historiques et dépolitisées. Le fait de constituer un discours secondaire leur donne l'occasion d'apparaitre comme des textes non idéologiques. Cependant, tout texte demeure idéologique.

$1[\ldots]$ these myths are not new, nor are they just myths, for they are also fairy tales. These myths and fairy tales are historically and culturally coded, and their ideological impact is great. Somehow they have become codified, authoritative, and canonical. We talk of classical myths and classical fairy tales. They seem to have been with us for centuries, for eternity, but we neglect the manner in which we created gods and magic to hold our experiences and lives intact (Traduction personnelle) 


\section{Copies, révisions et réécritures}

L'étude des formes comme les mythes suppose de se confronter au problème des versions. Toutefois, les débats sur la meilleure version doivent être évités en rejetant la hiérarchie traditionnelle privilégiant les originaux aux copies. En raison de l'origine orale des mythes, ce débat devient en quelque sorte insignifiant. Est-il possible de préférer un mythe «original» lorsqu'il nous est impossible de savoir quel est l'original ? Pourquoi un texte acquiert-il le statut d'original ? Ces questions nous amènent à réfléchir sur le rôle d'original / copie, surtout en ce qui concerne le statut conféré à chacun d'eux. Derrida (1997) consacre une partie de son travail au problème des discours secondaires. D'après lui, les copies n'occupent pas une position dans la hiérarchie, puisque les copies elles-mêmes créent l'original (l'original étant toujours différé, reporté). En outre, comme les copies sont les uniques formes écrites disponibles, elles doivent partager le même statut. La prétendue position secondaire s'en trouve rejetée.

Puisqu'on ne peut accéder qu'aux formes écrites, il faudrait accepter que le texte le plus ancien puisse être l'original. Cependant, cela n'est pas nécessairement le cas.

Que se passe-t-il quand un mythe est copié? Selon Zipes, cela signifie "to duplicate its message and images", mais dupliquer un mythe classique entraîne "to reproduce a set pattern of ideas and images that reinforce a traditional way of seeing, believing, and behaving» (Zipes, 1994: 9). Cependant, les copies ne sont jamais exactement comme les originaux, avant même la postmodernité: on sait que, même si un texte a été réécrit exactement à l'identique de ce qui a été publié, c'est un texte "différent» (Borges: «Pierre Menard, autor del Quijote», 1941). Les lectures précédentes du même texte rendraient impossible de le recevoir de la même manière qu'il a été reçu la première fois.

La révision des mythes est une révision des caractéristiques traditionnelles. Réécrire un mythe soulève toujours la possibilité de réinvention. Il met à jour l'histoire ancienne et l'adapte aux nouvelles conditions. À cet égard, Atwood affirme:

En muchos sentidos, los mitos no pueden ser realmente traducidos con propiedad desde su tierra natal, desde su propio lugar y tiempo. Nunca sabremos exactamente lo que significaron para sus antiguos públicos. Pero los mitos pueden usarse -como de hecho así ha sido con tanta frecuencia- 
como las piedras angulares para nuevas representaciones que encuentran sus significados dentro de sus propios tiempos y lugares. (Atwood, 2005: 58) ${ }^{2}$

C'est une forme littéraire qui doit être réécrite encore et encore. Chaque époque contribuera à ce que les mythes semblent «nouveaux", ils seront réécrits conformément aux nouvelles conditions et perspectives. Étant donné que chaque société doit produire ses propres mythes, il est absurde de maintenir que les mythes classiques sont mieux que les plus récents. Le privilège de l'original n'est plus applicable.

\section{Le mythe d'après Pedro Flores}

Les différentes versions du mythe de Thésée et le Minotaure ont été acceptées en tant que $\dot{\alpha} \lambda \dot{\eta} \theta \varepsilon \iota \alpha$ (aletheia). ${ }^{3}$ En ce sens, la proposition de Pedro Flores, qui connait parfaitement le mythe et ses versions littéraires ultérieures, montre une lecture différente, comme si ces versions officielles ne correspondaient pas à ce qui aurait pu arriver. À la base de ce livre de poèmes, le jeu et la relation entre la mémoire et l'oubli sont sous-jacents. Le point de vue des différents personnages, l'utilisation de la première personne, par exemple, pour raconter l'histoire elle-même, combinée à la troisième personne ou l'interpellation d'une deuxième personne, imposent une perspective polyédrique, éloignée, dans tous les cas, de ces mythes. Les personnages mythiques dans les vers de Flores reprennent vie afin de dire leur propre vérité, de montrer leur solitude et leur incompréhension, mais aussi leur ennui, et surtout, afin de sortir de la misère et la dégradation.

L'utilisation de la parodie et de l'ironie comme ressources rhétoriques dans ces poèmes se réfère à la poétique d'Aristote, qui porte sur les différences entre le genre historique et autres genres littéraires, mais aussi sur le problème de la vraisemblance. Aujourd'hui, au xxI ${ }^{\mathrm{e}}$ siècle, l'histoire est suspecte : ce qui a été traditionnellement accepté doit être mis en doute.

2 In many ways, myths cannot really be translated with any accuracy from their native soil -from their own place and time. We will never know exactly what they meant to their ancient audiences. But myths can be used -as they have been, so frequently- as the foundation stones for new renderings that find their meanings within their own times and places. (Traduction personnelle).

3 Les allusions les plus importantes au mythe de Thésée et le Minotaure se trouvent dans L'Odyssée; la Théogonie d'Hésiode, les Argonautiques d'Apollonios de Rhodes; dans la Bibliothèque Historique, de Diodore de Sicile; la Description de la Grèce, de Pausanias; chez Plutarque, dans l'une de ses vies consacrée à Thésée; Catulle, dans son carmen 64 et Ovide dans le chapitre huit des Métamorphoses, où l'on retrouve une lettre d'Ariane à Thésée, après que celui-ci l'eut abandonnée. 
C'est pourquoi, dans cette version poétique du mythe, tous les personnages impliqués en quelque sorte dans la version classique importent: Poséidon, Minos, Daedalus, Pasiphae, le taureau blanc, Héraclès, la mer Égée, les jeunes martyrs athéniens, Thésée, Ariane et, bien sûr, le Minotaure. Tous parmi eux entrent dans le domaine-espace du poème et, par le biais de ses fragments, il peut être entrevu une histoire complexe, dans laquelle tout n'est pas ce qui semble, où la vérité n'est pas ce qui a été raconté.

\section{Elenco}

Poseidón odia a Minos

por un toro más o menos

desangrándose en su santuario

frente al mar, en un crepúsculo rojizo.

La vanidad de Dédalo es tan susceptible

que Talos midió con sus gritos

la altura de la Acrópolis.

Hay un rastro de cuellos degollados

de allí de donde vienen las sandalias de Teseo.

Pasífae no duda en envenenar a cuanta mujer

se deja la piel en el lecho de Minos.

Egeo paga con carne de adolescentes

las derrotas de sus generales.

Minos cobra en carne de adolescentes

las victorias de sus generales.

Pero el mundo puede estar tranquilo:

la bestia consume sus días

en el fondo de un laberinto.

(Flores, 2012: 30)

Une œuvre aux semblables caractéristiques correspond mieux à la vie et la poésie et peut conduire à nous interroger sur notre lecture des mythes, sur le rôle qu'ils ont joué dans l'histoire. Le problème de la lecture est envisagé depuis Platon et Aristote, qui ont vu les mythes comme de la philosophie et les ont considérés comme un discours secondaire ou même marginal. Dans tous les cas, il est clair que le mythe a été déplacé à l'arrière-plan pendant lesdites transformations occidentales (de 1500 à 2000), qui ont établi le paradigme scientifique comme dominant. Le logos scientifique n'est pas compatible avec la conception mythique et le premier s'est imposé à la seconde. Cependant, et après une longue période durant laquelle les formes mythiques ont été reléguées au second plan, des écrivains, artistes et critiques ont revendiqué leur importance et y sont revenus en raison des possibilités que cette forme permettait. 
Le poète utilise une ressource commune: provoquer le malaise, tout d'abord parmi les personnages, puis parmi ses lecteurs. Les poèmes qui composent Donde príncipes y bestias essaient de dénaturer le mythe et démystifier la société dans laquelle il s'intègre, compte tenu des limites de confort formel et émotionnel. Il ne s'agit pas, cependant, d'une réécriture fermée, étrangère au monde actuel ou déconnectée de lui-même. Flores établit un dialogue avec différentes instances: tout d'abord, avec la tradition, avec les différentes versions du mythe et ses interprétations successives; en second lieu, avec des personnages historiques; en troisième lieu, avec d'autres textes littéraires et des auteurs avec lesquels il partage certaines caractéristiques. Ainsi, de la contemporanéité d'un touriste moderne à l'explicite référence de Borges, aussi bien dans le cas de la citation ouvrant le livre que dans la forme du personnage qui accompagne la plupart des protagonistes dans une hypothétique fin alternative, en passant par Edmond Dantès, le célèbre archéologue Schliemann ou les vers de Neruda, avec lesquels il y a un fructueux dialogue poétique, Pedro Flores construit un univers lui-même abordable, ouvert pour le lecteur, l'invitant à la réflexion et à la lecture.

Dans cette révision du mythe proposée par Flores, il y a une dualité que je tiens à souligner: celle du roi Minos et le Minotaure ou la bête, comme il est nommé à plusieurs reprises. Dans l'affrontement continu de ces deux personnages dans les poèmes, la misère du premier se manifeste contre l'authenticité et la liberté (excusez le paradoxe) du second, étant donné que le Minotaure, victime des caprices et des injustices des autres, reste toujours intègre, concentré comme il l'est sur son destin, innocent face à tout ce qui se produit. Ce n'est donc pas un hasard si le livre s'ouvre sur une citation de Borges: «¿De qué puedo quejarme? / En los atardeceres/ me pesa un poco la cabeza de toro» (Asterión dixit, c'est le nom du Minotauro). La citation appartient au poème "Quince monedas", dans La rosa profunda, publiée dans Obra poética [BB AA: Emecé, 1977, p. 430]. Immédiatement avant la citation on lit, dans le poème de Borges, le texte suivant: «El año me tributa mi pasto de hombres / y en la cisterna hay agua. / En mí se anudan los caminos de piedra». Ce n'est pas la seule fois que Borges visite le mythe de Thésée et le Minotaure: il le fait avec deux poèmes «El laberinto» et «Laberinto», et avec la nouvelle «La casa de Asterión»(El Aleph). C’est précisément la perspective de Borges à propos du Minotaure que Flores reprend dans son livre. En effet, il part de cette humanisation de la bête face à tout le monde pour raconter son histoire et, surtout, son désir de mourir des 
mains de Thésée, sachant que c'est son seul moyen, le seul repos possible qui lui reste. L'importance de cette perspective et de Borges est mise en évidence à différents moments, avec l'émergence de Borges qui apparaît comme un personnage supplémentaire. De cette façon, Flores intègre différentes lectures du mythe et joue toujours avec l'horizon d'attente de ses lecteurs.

La afrenta

Rey Minos:

el oprobio se crece en su prisión.

Tus enemigos se burlan

«la vergüenza del rey

tiene el tamaño de un templo».

El pueblo cruza apuestas

y los artistas dibujan al ser

cuyos gemidos parten el cielo de Creta.

Pero eres solo un aprendiz en el territorio milenario del rencor.

No sabías que bastaba abandonar a la criatura

por las abigarradas callejuelas de Cnossos,

que se topara con el asombro y el miedo,

con la incredulidad y el asco.

Con el verdadero y terrible laberinto:

ser distinto entre los hombres.

(Flores, 2012: 28)

El monstruo y los espejos

Para recordarle en cada momento que es distinto,

el rey ha llenado de espejos

los corredores que no llevan a sitio alguno,

las paredes en que desemboca la soledad.

El Minotauro pasa los días

mirándose en los espejos

para no olvidar, ni por un instante,

que es distinto al rey.

(Flores, 2012:15)

Le Minotaure, place donc son salut dans le fait d'être différent de l'autre, de se rappeler constamment que cette monstruosité n'est pas nécessairement déterminée par l’apparence extérieure.

\section{Le point du vue d'Ariane}

Il est une autre perspective remarquable dans le livre de poèmes: celle d'Ariane. Traditionnellement, elle a mis en évidence l'amour pour le héros et la trahison subséquente de Thésée en l'abandonnant à Naxos (bien que les versions varient, et que dans certaines le héros l'abandonne alors que dans 
d'autres ce soit un malheureux naufrage qui le conduise à Naxos), où Ariane retrouve Dionysos, avec qui elle part finalement.

Cette fois, Ariane tient les symboles, joue le rôle décisif dans l'histoire (tient le fil à l'entrée du labyrinthe afin que Thésée, qui tient l'autre bout du fil, puisse retrouver la sortie à la fin de sa mission). Mais qui donc attend Ariane à la sortie dans cette version du mythe proposée par Flores?

Ariadna

Ella sostiene el hilo a este lado del amor

esperando ver emerger del abismo

al dueño de su deseo,

a la carne de sus desvelos.

Con la espada bañada por la sangre del monstruo,

Teseo se postra a sus pies

y besa la luz de sus rodillas.

Ariadna no puede ocultar

una mueca de decepción. (Flores, 2012: 32)

Recuerdos de la abandonada de Naxos

Solo yo sabía el camino de regreso.

A veces te llevaba una caracola,

el color verde, un frasquito con viento.

Después de vencer al temor

el deseo no encuentra enemigo

en la estéril arquitectura de la venganza.

Te enseñé el idioma de las palabras

para que nombrando lo que nunca tendrías

fueras más rico que cualquier rey.

Cuando él llegó para matarte

le prometí que sería su reina.

Qué tenía distinto a los demás,

otro hombre con lengua de hombre

recitando mentiras de hombre,

otro héroe ensartado en tus astas.

Quién iba a pensar, amor mío,

que ese príncipe engreído y pálido

atravesara el lugar de tu pecho.

(Flores, 2012: 37)

Epitafio para Ariadna

Aquí yace Ariadna, princesa de Creta. 
Esta vez soy yo

la que surca la oscuridad

y lo desconocido.

Y nadie sostiene un hilo al otro lado. (Flores, 2012: 45)

Même dans l'épitaphe, Ariane prend la parole pour voir ce qui a toujours été une constante: la solitude.

Le livre de Pedro Flores, comme toute grande œuvre littéraire, offre différents niveaux de lecture. Grâce à une minutieuse mise en page et à la métrique des poèmes, "l'histoire» qui est configurée, avec une nouvelle formulation, ne nécessite pas une connaissance préalable du mythe pour pouvoir être comprise, car elle fonctionne parfaitement de façon autonome, en dehors de la tragédie ou de l'erreur. Après une première lecture, les personnages, d'une cohérence et d'une solidité remarquables, habitent depuis longtemps nos mémoires et nous rappellent que les princes et les bêtes ne sont pas si éloignés que l'on pourrait le penser, car ils se ressemblent trop entre eux.

Donde príncipes y bestias est un livre de poèmes qui séduit et invite à la relecture et s'enrichit à travers elle. En outre, si d'autres versions du mythe qui y est réécrit sont connues, le lecteur appréhendera les artifices de la langue, ce jeu soulevé par Flores, qui dose et élide l'information contenue dans ses poèmes, synthétise l'histoire par le biais de symboles et d'images, et rompt constamment l'horizon d'attente qui crée le mythe. C'est ainsi qu'il se présente dans le poème «El regreso improbable»:

El regreso improbable

Envuelto en una nube

de música y de risas

que hacen callar al viento,

un navío devora los raíles del agua.

Las velas no son blancas ni negras,

sino labradas con cien retales

que fueron faldas de otras tantas doncellas.

Los pájaros lamen los mástiles

salpicados de un vino joven.

En cubierta Ariadna retoza con la bestia

y el timón obedece a un bibliotecario ciego

que recita largas sagas islandesas.

Egeo decide que eso es más

de lo que un rey que se precie puede soportar

$\mathrm{y}$ de un salto bautiza el mar. 
¿No sería hermoso que por una vez

los príncipes no salieran victoriosos?

(Flores, 2012: 40)

La réécriture du mythe élaborée par Pedro Flores ne succombe pas à l'ordinaire ou à la tradition commune, elle offre une alternative à l'histoire traditionnelle, les "jeux de langages» privilégient d'une certaine manière ceux qui ont été systématiquement réduits au silence, comme le Minotaure, ou dont les motivations n'ont été jamais suffisamment expliquées, comme c'est le cas avec Ariane. Dans un monde où l'injustice est un fait quotidien, ce livre établit encore une fois et de façon différente l'histoire connue. Flores ne s'installe pas dans l'éloge de l'ordinaire ou de l'attendu et invente des raisons d'espérer, car, comme Flores l'écrit: «¿No sería hermoso que por una vez / los príncipes no salieran victoriosos?»

\section{Bibliographie}

Armstrong, K., 2005, A Short History of Myth, Edinburgh, Canongate.

Atwood, M., 2005, "The Myth Series and Me», en Publishers Weekly, vol. 252, n 47 p. 58.

Barthes, R., 2000, Mythologies, London, Vintage orig. in French, 1957.

Bettelheim, B., 1991, The Uses of Enchantment. The Meaning and Importance of Fairy Tales, London, Penguin.

Borges, J.L., 1977, Obra poética. Buenos Aires, Emecé.

Brunel, P., 1992, Mythocritique. Théorie et parcours. Paris, PUF.

Derrida, J., 1997, Of Grammatology, Trans. Gayatri Chakravorty Spivak, Baltimore \& London, The Johns Hopkins University Press.

Flores, P., 2012, Donde príncipes y bestias, Granada, Tragacanto.

Todorov, T., 1987, «L'origine des genres », in La notion de littérature et autres essais, Paris, Seuil.

Zipes, J., 1994, Fairy Tales as Myth, Myth as Fairy Tales, Kentucky, The University Press of Kentucky. 